\title{
Efecto de los Probióticos en las Condiciones Periodontales
}

\author{
Effect of Probiotics on Periodontal Conditions
}

\author{
Muñoz Salas K¹, Alarcón Palacios M²
}

\begin{abstract}
RESUMEN
Los probióticos se definen como microorganismos que, administrados en cantidades adecuadas, brindan un beneficio en la salud del huésped. El efecto del tratamiento con probióticos viene siendo estudiado extensamente en una diversidad de indicaciones sistémicas y desórdenes médicos. Recientemente existe un gran interés en el control probiótico contra las infecciones orales más comunes como la caries dental y la periodontitis, sin embargo, la información disponible sobre los efectos de probióticos en la salud periodontal y sus condiciones clínicas es aún limitada. La presente revisión describe el conocimiento actual en bacterioterapia con probióticos desde una perspectiva en la salud oral y periodontal.
\end{abstract}

Rev. Clin. Periodoncia Implantol. Rehabil. Oral Vol. 3(3); 136-139, 2010.

Palabras clave: Probióticos, salud periodontal, bacterioterapia, terapia de reemplazo.

\begin{abstract}
Probiotics are defined as live microorganisms that administered in adequate amounts, bring beneficial effect on the health of the host. The effect of probiotic therapy has been studied extensively for a variety of systemic indications and medical disorders. Recently, there has been increasing interest in probiotic for the control of the most common oral infectious diseases such as dental caries and periodontitis. However, only limited information is available regarding the effect of probiotics on periodontal health and clinical conditions. This review describes current knowledge on probiotic bacteriotherapy from the oral health and periodontal perspective.
\end{abstract}

Rev. Clin. Periodoncia Implantol. Rehabil. Oral Vol. 3(3); 136-139, 2010.

Key words: Probiotics, periodontal health, bacteriotherapy, replacement therapy.

\section{INTRODUCCIÓN}

La resistencia antibiótica con la emergencia de cepas multiresistentes es un problema global que va en aumento; este desarrollo lamentable ha llevado a los científicos a buscar otras formas de combatir las enfermedades infecciosas ${ }^{(1,2)}$. Un nuevo método, el enfoque probiótico, es investigado con respecto a la eliminación de los microorganismos patogénicos. Los probióticos son microorganismos vivos, los cuales, administrados en cantidades adecuadas, brindan un beneficio en la salud del huésped ${ }^{(3)}$. Las bacterias probióticas pueden ocasionar beneficios en la salud del huésped brindándole nutrientes y cofactores, compitiendo directamente con los patógenos, interactuando con los factores de virulencia de éstos y estimulando la respuesta inmune del huésped ${ }^{(4)}$. El efecto del tratamiento con probióticos ha sido estudiado extensamente para una diversidad de indicaciones sistémicas y desórdenes médicos. Por ejemplo, los beneficios potenciales de los probióticos han sido estudiados en desórdenes gastrointestinales ${ }^{(4,6)}$, ginecológicos y eczema ectópico ${ }^{(7,8)}$. Recientemente hay un interés creciente en el control probiótico contra las infecciones orales.

Las infecciones orales constituyen las formas más comunes de las infecciones en humanos ${ }^{(9)}$. La cavidad oral es un ecosistema complejo en el cual se desarrolla una microbiota diversa. El amplio rango de $\mathrm{pH}$, la disponibilidad de nutrientes, las superficies deslizantes y no deslizantes, la saliva, los fluidos creviculares y las comunidades microbianas fluctúan en composición y en actividad metabólica pero llegan a una especie de homeostasis con el huésped. Los cambios en el ambiente, ya sean por enfermedad, conductas, dieta o medicamentos, alteran la homeostasis y permiten las infecciones endógenas o la susceptibilidad a las infecciones exógenas. La microflora oral residente es diversa y está compuesta por especies con diferentes requerimientos nutricionales (sacarolíticos, proteolíticos, consumidores secundarios), atmosféricos (aeróbicos, anaeróbicos, facultativos, microaerofílicos, capnofílicos) y físico-químicos $(\mathrm{pH}, \mathrm{co} \text {-factores })^{(10)}$. Las enfermedades dentales son una consecuencia de los cambios en la ecología señalada anteriormente. Si el ambiente local es perturbado, entonces los patógenos potenciales pueden ganar una ventaja competitiva y, bajo condiciones apropiadas, alcanzar números que predisponen a enfermedad. Presumiblemente la administración oral de probióticos puede beneficiar la salud oral previniendo el crecimiento de la microbiota nociva o modulando la inmunidad de la mucosa en la cavidad oral.

Los estudios de los potenciales probióticos orales se han enfocado en la prevención de la caries, especialmente en la posibilidad de reducir el número de Streptococcus mutans cuando se usan productos conteniendo ciertas cepas de probióticos ${ }^{(11,12)}$. También se han investigado otras posibles aplicaciones, como la reducción del número de Cándida oral ${ }^{(13)}$ o de la microflora asociada con la halitosis ${ }^{(14,15)}$. Sin embargo, existe limitada información disponible con respecto al efecto de los probióticos en la salud periodontal y sus condiciones clínicas.

A continuación se desarrollan los conceptos generales de los probióticos y el conocimiento actual de la bacterioterapia con ellos desde una perspectiva de la salud oral y sobre todo de los efectos en la salud periodontal y sus condiciones clínicas.

1. Residente. Postgrado Periodoncia e Implantes. Universidad Peruana Cayetano Heredia. Perú.

2. Docente. Postgrado Periodoncia e Implantes. Universidad Peruana Cayetano Heredia. Perú. 


\section{PROBIÓTICOS}

El término probiótico proviene del término griego que significa

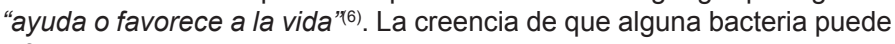
influenciar en la salud data de los principios del siglo XX, cuando el premio Nobel Elie Metchnikoff, de origen ruso (1907), reportó que los búlgaros vivían mucho más que los ciudadanos de otras naciones y supuso que se debía al consumo de productos lácteos fermentados que contenían bacterias. La idea era que la bacteria en los productos fermentados, competía con los microorganismos que eran dañinos para la salud ${ }^{(9)}$.

Metchnikoff trabajó en el Instituto Pasteur en París y descubrió el Lactobacillus bulgaricus, una cepa que más tarde introduciría en la producción comercial de productos ácido-lácteos en Francia y toda Europa. El invirtió la última década de su vida al estudio de esta bacteria, como una forma de aumentar la longevidad humana. Fue entonces que nació el concepto probiótico y se abrió un nuevo campo en la microbiología ${ }^{(16)}$.

Lilly y Stillwel(17) (1965), quienes propusieron el término probiótico como un antónimo del término antibiótico, fueron los primeros que describieron los probióticos como ciertos microorganismos vivos no patógenos que incluían alguna flora bacteriana, la cual tiene un efecto benéfico en la salud del huésped y en la prevención de la enfermedad y/o sirven como tratamiento. Debido al aumento de investigaciones en las ciencias básicas y estudios clínicos, la Organización de Alimentos y Agricultura de las Naciones Unidas (FAO - Food and Agriculture Organization) y la Organización Mundial de la Salud (WHO - World Health Organization) definieron el término probiótico como "microorganismos vivos, los cuales, cuando son consumidos en cantidades adecuadas como parte de los alimentos, brindan un beneficio en la salud del huésped"(3,6,18). Aunque últimamente se pueden considerar algunas modificaciones en la definición, debido a los resultados de las nuevas investigaciones en las cuales se observó que los microorganismos inactivados o sus componentes celulares también pueden influir en la salud humana, dichos hallazgos pueden ampliar el concepto de probióticos restando importancia a los microorganismos vivos ${ }^{(19)}$.

Las primeras especies de probióticos introducidas en las investigaciones fueron: el Lactobacillus acidophilus por Hull y col. (1984) y el Bifidobacterium bifidum por Holcombh y col. (1991)(9).

Un número de cepas bacterianas han sido aisladas y estudiadas con el objetivo de un uso clínico. Los probióticos más comúnmente usados y estudiados son los Lactobacillus spp. y los Bifidobacterium spp., y una de sus características generales incluye su origen humano ${ }^{(16)}$. Sin embargo, todos los probióticos no tienen la misma eficacia. Es necesario definir bien las potenciales cepas probióticas antes de su uso.

En algunos países el crecimiento de la industria en la producción de los probióticos hace difícil comprar yogurts que no contengan bacterias probióticas.

Los fabricantes de los cultivos recomiendan la formulación de estos productos con una cantidad de 106 bacterias probióticas por gramo o milímetro de productos diarios, pero los conteos viables pueden caer por debajo de estos niveles, especialmente al final de su periodo de validez. Definidos como "probióticos médicos" (preparación microbiana) y "otros probióticos" (alimentos funcionales), los probióticos se ofrecen en productos en cuatro formas básicas ${ }^{(9)}$ :

- Como un cultivo concentrado añadido a una bebida o a un alimento (como un jugo de fruta).

- Inoculado en fibras prebióticas (ingredientes no digestibles que se encuentran en los alimentos que favorecen el crecimiento de los probióticos).

- Inoculado en alimentos lácteos (productos de consumo diario como leche, yogurt y queso).

- Como concentrado y envasado como suplementos dietéticos (productos que no son de consumo diario como cápsulas, tabletas de gelatina, presentación en polvo, gomas de mascar y gotas $)^{(1,33)}$.

\section{EFECTO DE LOS PROBIÓTICOS EN LA SALUD ORAL}

Gorbach y Goldin (1985) aislaron del intestino humano el Lactobacillus rhamnosus GG (LGG) que es la bacteria probiótica más ampliamente estudiada ${ }^{(16)}$. Se demostró que produce una sustancia con un potencial inhibitorio contra la actividad de diferentes especies bacterianas, incluyendo las especies cariogénicas como los Streptococcus spp. ${ }^{(21)}$.

Näse y col. (2001), en un estudio en niños de 1 a 6 años, en Finlandia, examinaron Lactobacillus rhamnosus GG: LGG en leche, y se encontró que reduce significativamente el riesgo de caries. Se concluyó que el uso de leche que contenga la bacteria probiótica $L G G$ brinda efectos benéficos en la salud oral de esos niños ${ }^{(9)}$.

Grudianov y col. (2002) encontraron que los probióticos se adhieren al tejido dental, establecen un efecto cariostático y pueden formar parte del biofilm que lucha contra las bacterias cariogénicas ${ }^{(9)}$.

Ahola y col. (2002) determinaron que la intervención con probióticos: $L G G$ y Bifidobacterium spp. reduce el riesgo de elevados niveles de Streptococcus mutans ${ }^{(9)}$.

Wei y col. (2002) produjeron altas concentraciones de anticuerpos contra las bacterias cariogénicas humanas: Streptococcus mutans y Streptococcus sobrinus, en calostro de leche fermentada de bovino $^{(22)}$.

Vancikova y col. (2003), basándose en el estudio anterior, observaron que los probióticos mejoran la respuesta oral inmune ${ }^{(9)}$.

Von Bultzingslowen y col. (2003) determinaron que cepas probióticas selectas como $L$. plantarum refuerzan la cavidad oral y el tracto gastrointestinal mediante una diseminación bacteriana, lo cual mejora la ingesta de alimentos y el peso corporal en animales a los cuales se les realizó quimioterapia. Esto tiene un efecto positivo en el campo de la oncología, ya que durante la quimioterapia, en casos de cáncer, se pueden presentar infecciones sistémicas por una alteración en la microflora orofaríngea y gastrointestinal, causando inmunosupresión ${ }^{(23)}$.

Hatakka y col. (2005), en un estudio en adultos mayores, los cuales consumieron queso probiótico, mostraron que los probióticos reducen la prevalencia de Candida spp. oral, y también la hiposalivación. Esta observación es interesante teniendo en cuenta el incremento de la resistencia a las drogas de la Candida spp. ${ }^{(24)}$.

\section{EFECTO DE LOS PROBIÓTICOS EN LAS CONDICIONES PERIODONTALES}

Las enfermedades periodontales, junto a la caries dental, representan la mayor parte de las enfermedades orales. Según la Organización Mundial de la Salud, la mayoría de los niños presentan signos de gingivitis y entre los adultos las etapas iniciales de la enfermedad periodontal son altamente prevalentes ${ }^{(19)}$.

El biofilm bacteriano, que se forma en los tejidos duros y blandos de la cavidad oral, es considerado como el principal factor etiológico en la mayoría de las condiciones patológicas de la cavidad oral. La acumulación de bacterias dentro del biofilm, facilitado por un pobre mantenimiento de la salud oral, predispone a cambios en la comunidad microbiana, conduciendo a la aparición de la inflamación periodontal(20). La complejidad de la microbiota periodontal se asemeja a la microbiota del tracto gastro-intestinal, donde las enfermedades infecciosas son tratadas mediante probióticos. En la cavidad oral, la terapia de reemplazo muestra beneficios en la prevención de la caries dental(21), aunque aún existen pocos estudios que muestren la efectividad del uso de probióticos en el tratamiento de la periodontitis. En la Tabla 1 se resume las especies probióticas usadas para aplicación oral en general.

Teniendo en cuenta las dos mayores estrategias contra las enfermedades periodontales: la eliminación de patógenos específicos y la supresión de la respuesta destructiva del huésped, el enfoque de la terapia con probióticos puede ser de utilidad para lograr el objetivo de estos tratamientos ${ }^{(19)}$.

Grudianov y col. (2002) examinaron tabletas probióticas con Bifidobacterium spp. en un tratamiento de gingivitis y diferentes grados de periodontitis. El tratamiento de los pacientes del grupo control fue con "Tantum verde". Los casos de gingivitis y periodontitis fueron tratados con probióticos, el efecto de ellos para la normalización de la microflora fue mayor en comparación con el grupo que recibió Tantum verde ${ }^{(9,25)}$.

Ishikawa (2003) demostró que cepas de Lactobacillus spp. son útiles en la reducción de la inflamación gingival y en el número de bacilos pigmentados como Porphyromonas gingivalis, en saliva y placa subgingival(26).

Burton y col. (2005) concluyeron que las cepas bacterianas probióticas originarias de la microbiota oral de humanos sanos, tienen una aplicación potencial para la prevención y tratamiento de la halitosis. En este estudio se empleó el Streptococcus salivarius, el cual según 
Tabla 1. Especies de probióticos y su efecto en la salud en la cavidad oral (resultado de investigaciones clínicas).

\begin{tabular}{|c|c|c|c|}
\hline $\begin{array}{l}\text { Especies de } \\
\text { Probióticos }\end{array}$ & Efecto & $\begin{array}{c}\text { Medios de } \\
\text { Administración }\end{array}$ & Referencia \\
\hline $\begin{array}{l}\text { L. reuteri ATCC } 55730 \\
\text { L. reuteriATCCPTA5289 }\end{array}$ & $\begin{array}{l}\text { Reducción en los niveles } \\
\text { salivales de S. mutans }\end{array}$ & Pastillas & Caglar et al. 2008 \\
\hline L. reuteri ATCC 55730 & Reducción de S. mutans & Tabletas & Caglar et al. 2006 \\
\hline $\begin{array}{l}\text { L. mamnosus GG, } \\
\text { ATCC } 53103\end{array}$ & $\begin{array}{l}\text { Reducción del riesgo de } \\
\text { niveles elevados de } \\
\text { S. mutans }\end{array}$ & Queso & \\
\hline $\begin{array}{l}\text { L. mamnosus GG, } \\
\text { ATCC } 53103\end{array}$ & $\begin{array}{l}\text { Reducción en los niveles } \\
\text { salivales de S. mutans }\end{array}$ & Leche & \\
\hline $\begin{array}{l}\text { Bifidobacterium } \\
\text { DN-173010 }\end{array}$ & $\begin{array}{l}\text { Supresión del crecimiento } \\
\text { de bacterias negras } \\
\text { pigmentadas en } \\
\text { muestras de saliva } \\
\text { implicadas con halitosis }\end{array}$ & Yogurt & \\
\hline S. salivanius $\mathrm{K} 12$ & $\begin{array}{l}\text { Reducción de gingivitis } \\
\text { y placa }\end{array}$ & $\begin{array}{l}\text { Enjuagatorios } \\
\text { bucales }\end{array}$ & \\
\hline L. reuteri & & $\begin{array}{l}\text { Formulación } \\
\text { probiótica }\end{array}$ & \\
\hline L. casei 37 & & $\begin{array}{l}\text { Apósito } \\
\text { periodontal }\end{array}$ & \\
\hline $\begin{array}{l}\text { L. mamnosus GG(ATCC } \\
\text { 53103), L. mamnosus } \\
\text { LC705, Propionibactenium } \\
\text { freudenreichii ssp., } \\
\text { shermanii JS }\end{array}$ & $\begin{array}{l}\text { Mejora de periodontitis } \\
\text { en relación a signos y } \\
\text { síntomas }\end{array}$ & Queso & \\
\hline L. acidophilus LAFTIL10 & $\begin{array}{l}\text { Mejora de los signos } \\
\text { clínicos periodontales en } \\
\text { fumadores y no } \\
\text { fumadores }\end{array}$ & & \\
\hline
\end{tabular}

Kazor y col. (2003) es considerado como un colonizador temprano y predominante de la microbiota de la lengua en individuos sanos, y según Yoshida y col. (2003), tiene sólo una limitada capacidad para producir compuestos volátiles de sulfuro(27).

Kang (2006) aisló Weissella cibaria en niños sanos entre 4 y 7 años, y fue aplicado en la cavidad oral de individuos sanos entre 20 y 30 años. Los resultados mostraron que $W$. cibaria inhibe la producción de los compuestos volátiles de sulfuro responsables de la halitosis, inhibiendo la proliferación de F. nucleatum, el cual es eliminado por el peróxido de hidrógeno producido por W. cibaria ${ }^{(28)}$.

Krasse y col., Matsuoka y col. (2006) mostraron la disminución del sangrado gingival y la reducción de la gingivitis mediante la administración de Lactobacillus Reuteri(18).

Teughels y col. (2007), en un estudio realizado en perros beagle, en defectos óseos creados, muestra la aplicación subgingival de bacterias benéficas como una terapia adjunta luego del pulido radicular (no se aplicó terapia antibiótica), lo que se denominó Recolonización Guiada de la bolsa, esto aumentaría la carga microbiana benéfica en las bolsas periodontales, impidiendo la recolonización con los periodontopatógenos. Esta técnica no excluyó totalmente los periodontopatógenos, pero sí retardó el proceso de recolonización significativamente. Esta técnica prueba la teoría del uso de probióticos en la recolonización tardía del surco periodontal en un modelo animal y se podría considerar como una alternativa de tratamiento válida para la periodontitis en vista de la creciente resistencia antibiótica ${ }^{(29)}$.

Shimauchi y col. (2008), en un estudio a doble ciego, en pacientes fumadores y no fumadores que no presentaban periodontitits severa (se consideraron así dientes con $P S \geq 6 \mathrm{~mm}$, con movilidad excesiva y abscesos), tomó dos grupos al azar y administró tabletas conteniendo Lactobacillus salivarius WB2 y xilitol en un grupo y placebo (xilitol) en el otro, encontrando que la administración oral del probiótico Lactobacillus salivarius WB21 disminuye significativamente el índice de placa y la profundidad de bolsa de los pacientes fumadores, lo que sugiere una mejoría clínica de las condiciones periodontales mediante el uso del probiótico. También se observó una diferencia significativa de los niveles salivales de lactoferrina, la cual está correlacionada con los parámetros clínicos de periodontitis, a las ocho semanas para los pacientes fumadores; concluyendo así que los probióticos son útiles para la mejora y mantenimiento de la salud oral en los pacientes con un alto riesgo de enfermedad periodontal ${ }^{(26)}$

Mayanagi y col. (2009), en un estudio donde se administró en forma oral Lactobacillus salivarius WB21 a pacientes con periodontitis, muestra que se redujo la cantidad de cinco bacterias periodontopatógenas: Aggregatibacter actinomycetemcomitans, Prevotella intermedia, Porphyromonas gingivalis, Treponema denticola y Tanarella forsythia, lo cual puede contribuir a mejorar las condiciones periodontales, incluyendo la profundidad de bolsa, el índice de placa, el índice gingival y la disminución del sangrado(18).

Van Essche y col. (2009), en un estudio in vitro en condiciones semejantes a las de la cavidad oral, demostraron que la cepa bacteriana Bdellovibrio bacteriovorus HD100 puede atacar, alimentarse y matar al Aggregatibacter actinomycetemcomitans, lo que nos demuestra su potencial como un antibiótico viviente, en la prevención y el tratamiento de la periodontitis. Bdellovibrio spp. son proteobacterias altamente móviles que se alimentan de bacterias Gramnegativas, están presentes en toda la naturaleza y han sido aislados de diferentes hábitats terrestres y acuáticos, de biofilms, así como de heces de animales( ${ }^{(30)}$. El Aggregatibacter actinomycetemcomitans es considerado como un periodontopatógeno clave y está asociado con la periodontitis agresiva(31). La periodontitis asociada con Aggregatibacter actinomycetemcomitans es difícil de tratar únicamente mediante la remoción mecánica del biofilm subgingival, por lo que es necesario el tratamiento complementario con antibióticos sistémicos ${ }^{(32)}$. De ahí la importancia de este estudio que muestra una alternativa diferente a la antibioterapia $^{(30)}$.

\section{CONCLUSIONES}

En un esfuerzo por reducir el uso de antibióticos, y en vista del creciente desarrollo de la resistencia bacteriana a estos, la Organización Mundial de la Salud respalda, en lo posible, el uso de probióticos como una terapia de interferencia microbiana, apoyando el uso de microorganismos no patógenos para eliminar los patógenos.

Las investigaciones clínicas muestran los efectos positivos en la salud humana asociada al uso de probióticos. Existen evidencias científicas que confirman sus potenciales benéficos en condiciones como las infecciones gastrointestinales, genitourinarias, alergias, desórdenes de colon y hasta cáncer.

Es por esto que también se considera el empleo de probióticos como una alternativa de tratamiento válida en el control de las enfermedades periodontales, ya que mejora las condiciones del huésped reduciendo la profundidad de la bolsa, la inflamación, el sangrado gingival y la halitosis.

Sin embargo, es un campo nuevo aún con respecto a la salud oral, específicamente periodontal; por esto se necesitan más estudios que confirmen el control biológico de la placa mediante el uso de probióticos como una alternativa de tratamiento en el control de las enfermedades periodontales.

También es interesante la idea de reemplazar los organismos dañinos con bacterias inactivadas o modificadas que contengan características beneficiosas, por lo que sería necesario realizar investigaciones en el campo de los probióticos. 


\section{REFERENCIAS BIBLIOGRÁFICAS}

1. Beers $M H$, Berkow R, eds. Infectious diseases: antibacterial drugs. In: The Merck Manual, $17^{\text {th }}$ ed. Whitehouse Station: Merck Research Laboratories, 1999; 1101-1127.

2. Costerton JW, Stewart PS, Greenberg EP. Bacterial biofilms: a common cause of persistent infections. Science, 1999; 284: 1318-1322.

3. FAO/WHO. Report of joint FAO/WHO expert consultation on evaluation of health and nutritional properties of probiotics in food including powder milk with live lactic acid bacteria. 2001; Córdoba, Argentina.

4. Saier MH Jr., Mansour NM. Probiotics and prebiotics in human health. Journal of Molecular Microbiology and Bio-technology, 2005; 10: 22-25.

5. Vanderhoof JA, Whitney DB, Antonson DL, Hanner TL, Lupo JV, Young RJ. Lactobacillus $G G$ in the prevention of antibiotic-associated diarrhea in children. The Journal of Pediatrics, 1999; 135: 564-568.

6. Vanderpool MD, Fan Yan MD PhD, D Brent Polk MD. Mechanisms of probiotic action: implications for therapeutic applications in inflammatory bowel diseases. Inflamm Bowel Dis, Vol. 14, II November 2008.

7. McLean NW, Rosenstein IJ. Characterisation and selection of a Lactobacillus species to re-colonise the vagina of women with recurrent bacterial vaginosis. Journal of Medical Microbiology, 2000; 49: 543-552.

8. Reid G, Charbonneau D, Erb J, Kochanowski B, Beuerman D, Poehner $\mathrm{R}$, Bruce AW. Oral use of Lactobacillus rhamnosus GR-1 and L. fermentum RC-14 significantly alters vaginal flora: randomized, placebo-controlled trial in 64 healthy women. FEMS Immunology and Medical Microbiology, 2003; 35: 131-134

9. Caglar E, Kargul B, Tanboga I. Bacteriotherapy and probiotics role on oral health. Oral Diseases, 2005; 11: 131-137.

10. Cassell G, Ellen R, Mangan DF et al. Infectious diseases planning, Workshop Report. National Institute of Dental \& Craniofacial Research: Bethesda, MD 2005.

11. Twetman S, Stecksén-Blicks C. Probiotics and oral health effects in children. International Journal of Pediatric Dentistry, 2008; 18: 3-10.

12. Anderson M, Shi W. A probiotic approach to caries management. Pediatr Dent, 2006; 28(2): 151-153.

13. Elahi S, Pang G, Clancy A, Clancy R. Enhanced clearance of Candida albicans from the oral cavities of mice following oral administration of Lactobacillus acidophilus. Clin Exp Immunol, 2005; 141: 29-36.

14. Kang MS, Kim BG, Chung J, Lee HC, Oh JS. Inhibitory effect of Weissella cibaria isolates on the production of volatile sulphur compound. J Clin Periodontol, 2006; 22: 226-232.

15. Burton JP, Chilcott CN, Moore CJ, Speiser G, Tagg JR. A preliminary study of the effect of probiotic Streptococcus salivarius K12 on oral malodour parameters. J Appl Microbiol, 2006; 100: 754-764.

16. Meurman JH. Probiotics: do they have a role in oral medicine and dentistry? Eur J Oral Sci, 2005; 113: 188-196.

17. Lilly DM, Stillwell RH. Probiotics: growth-promoting factors produced by microorganisms. Science, 1965; 147: 747-748.

18. Mayanagi G, Kimura M, Nakaya S, Hirata H, Sakamoto M, Benno $Y$, Shimauchi $H$. Probiotic effects of orally administered Lactobacillus salivarius WB21-containing tablets on periodontopathic bacteria: a doubleblinded, placebo-controlled, randomized clinical trial. J Clin Periodontol, 2009; 36: 506-513.
19. Stamatova I, Meurman J. Probiotics and periodontal disease. Periodontol 2000, 2009; 51: 141-151.

20. Newman $\mathrm{H}$. Plaque and chronic inflammatory periodontal disease. A question of ecology. J Clin Periodontol, 1990; 17: 533-541.

21. Haukioja A, Loimaranta V, Tenovuo J. Probiotic bacteria affect the composition of salivary pellicle and streptococcal adhesion in vitro. Oral Microbiol Immunol, 2008; 23: 336-334.

22. Wei H, Loimaranta V, Tenovuo J. Stability and activity of specific antibodies against Streptococcus mutans and Streptococcus sobrinus in bovine milk fermented with Lactobacillus rhamnosus strain GG or treated at ultra-high temperature. Oral Microbiol Immunol, 2002; 17: 9-15.

23. Von Bultzingslowen I, Adlerberth I, Wold AE. Oral and intestinal microflora in 5-fluorouracil treated rats, translocation to cervical andmesenteric lymph nodes and effects of probiotic bacteria. Oral Microbiol Immunol, 2003; 18: 278-284.

24. Hatakka K, Ahola A, Yli-Knuuttila H, Richardson M, Poussa T, Suomalainen T, Meurman JH, Korpela R. Cheese with probiotic bacteria reduces prevalence of oral Candida in the elderly. Am J Clin Nutr, 2005; in press.

25. Grudianov Al, Dimitrieva NA, Fomenko EV. Use of probiotics Bifidumbacterin and Acilact in tablets in therapy of periodontal inflammations. Stomatologiia Mosk, 2002; 81: 39-43.

26. Shimauchi H, Mayanagi G, Nakaya S, Minamibuchi M, Ito Y, Yamaki $\mathrm{K}$, Hirata $\mathrm{H}$. Improvement of periodontal condition by probiotics with Lactobacillus salivarius WB21: a randomized, double-blind, placebocontrolled study. J Clin Periodontol, 2008; 35: 897-905.

27. Burton JP, Chilcott CN, Tagg JR. The rationale and potential for the reduction of oral malodour using Streptococcus salivarius probiotics. Oral Diseases, 2005; 11(Suppl 1): 29-31.

28. Kang MS, Kim BG, Chung J, Lee HC, Oh JS. Inhibitory effect of Weissella cibaria isolates on the production of volatile sulphur compounds. J Clin Periodontol, 2006; 33: 226-232.

29. Teughels W, Newman MG, Coucke W, Haffajee AD, Van Der Mei HC Kinder Haake S, Schepers E. Guiding periodontal pocket recolonization: a proof of concept. J Dent Res, 2007; 86(11): 1078-1082.

30. Van Essche, Quirynen, Sliepen I, Van Eldere J, Teughels. Bdellovibrio bacteriovorus attacks Aggegatibacter actinomycetemcomitans. J Dent Res, 2009; 88(2): 182-186.

31. Tonetti MS, Mombelli A. Early-onset periodontitis. Ann Periodontol, 1999; 4: 39-53.

32. Herrera D, Sanz M, Jepsen S, Needleman I, Roldán S. A systematic review on the effect of systemic antimicrobials as an adjunct to scaling and root planing in periodontitis patients. J Clin Periodontol, 2002; 29(Suppl. 3): 136-159.

33. http://www.biogaia.com/ 